\title{
Existence of fast homoclinic solutions for a class of second-order damped vibration systems
}

\section{Qiongfen Zhang ${ }^{1 *}$}

\section{"Correspondence: \\ qfzhangcsu@163.com \\ ${ }^{1}$ College of Science, Guilin \\ University of Technology, Guilin, P.R. China}

\begin{abstract}
By applying the mountain pass theorem in critical point theory, the existence of fast homoclinic solutions is obtained for the following second-order damped vibration system:

$$
\ddot{u}(t)+q(t) \dot{u}(t)-L(t) u(t)-a(t)|u(t)|^{p-2} u(t)+\nabla W(t, u(t))=0,
$$

where $p \in(2,+\infty), t \in \mathbb{R}, u \in \mathbb{R}^{N}, L(t)$ is a positive definite symmetric matrix-valued function for all $t \in \mathbb{R}, W \in C^{1}\left(\mathbb{R} \times \mathbb{R}^{N}, \mathbb{R}\right)$ is not periodic in $t, a(t)$ is a continuous, positive function on $\mathbb{R}$ and $q: \mathbb{R} \rightarrow \mathbb{R}$ is a continuous function and $Q(t)=\int_{0}^{t} q(s) d s$ with $\lim _{|t| \rightarrow+\infty} Q(t)=+\infty$.
\end{abstract}

MSC: $34 C 37 ; 35 A 15 ; 37 J 45 ; 47 J 30$

Keywords: Fast homoclinic solutions; Mountain pass theorem; Existence; Critical point

\section{Introduction}

Consider fast homoclinic solutions of the following second-order system:

$$
\ddot{u}(t)+q(t) \dot{u}(t)-L(t) u(t)-a(t)|u(t)|^{p-2} u(t)+\nabla W(t, u(t))=0,
$$

where $p \in(2,+\infty), t \in \mathbb{R}, u \in \mathbb{R}^{N}, L(t)$ is a positive definite symmetric matrix-valued function for all $t \in \mathbb{R}, W \in C^{1}\left(\mathbb{R} \times \mathbb{R}^{N}, \mathbb{R}\right)$ is not periodic in $t, a(t)$ is a continuous, positive function on $\mathbb{R}$, and $q: \mathbb{R} \rightarrow \mathbb{R}$ is a continuous function and $Q(t)=\int_{0}^{t} q(s) d s$ with

$$
\lim _{|t| \rightarrow+\infty} Q(t)=+\infty
$$

When $q(t) \equiv 0$ and $L(t) \equiv 0$, problem (1.1) reduces to the following special second-order Hamiltonian system:

$$
\ddot{u}(t)-a(t)|u(t)|^{p-2} u(t)+\nabla W(t, u(t))=0, \quad \text { a.e. } t \in \mathbb{R} \text {. }
$$

(c) The Author(s) 2018. This article is distributed under the terms of the Creative Commons Attribution 4.0 International License (http://creativecommons.org/licenses/by/4.0/), which permits unrestricted use, distribution, and reproduction in any medium, provided you give appropriate credit to the original author(s) and the source, provide a link to the Creative Commons license, and indicate if changes were made. 
In [1, 2], and [3], the authors considered homoclinic solutions for the special Hamiltonian system (1.3) in a weighted Sobolev space and obtained some results by using the mountain pass theorem in critical point theory. For the applications of mountain pass theorem, please see the references [4] and [5]. In [6], Benci and Fortunato investigated a class of nonlinear Dirichlet problems in a weighted Sobolev space.

When $p=2$ and $L(t) \equiv 0$, problem (1.1) reduces to the following nonlinear second-order damped vibration problem:

$$
\ddot{u}(t)+q(t) \dot{u}(t)-a(t) u(t)+\nabla W(t, u(t))=0, \quad \text { a.e. } t \in \mathbb{R} .
$$

When $a(t) \equiv 0$ and $q(t) \equiv 0$, problem (1.1) becomes the following second-order Hamiltonian system:

$$
\ddot{u}(t)-L(t) u(t)+\nabla W(t, u(t))=0, \quad \text { a.e. } t \in \mathbb{R} \text {. }
$$

As we known, the existence of homoclinic orbits is very important in the study of the behavior of dynamical systems. The first work about homoclinic orbits was done by Poincaré [7].

In the past years, the existence and multiplicity of homoclinic solutions for system (1.5) were investigated by many researchers by using critical point theory. For example, see [819], and the references cited therein. For the existence of homoclinic solutions for damped vibration problem (1.4), please see the literature [20-25], and the references cited therein. For other kinds of damped vibration problem, please see the literature [26] and [27]. Besides, by applying the mountain pass theorem and symmetric mountain pass theorem in critical point theory, Zhang and Li [28] investigated the existence and multiplicity of fast homoclinic solutions for a class of nonlinear second-order nonautonomous systems and obtained some results, which generalized and improved problem (1.4).

Motivated mainly by the above mentioned works, we will investigate fast homoclinic solutions for problem (1.1) and establish some results. In the following, we first state some properties of the weighted Sobolev space $E$ and then introduce the concept of fast homoclinic solutions for problem (1.1). On the weighted Sobolev space $E$, a certain variational functional associated with (1.1) is defined and fast homoclinic solutions are the critical points of the certain functional.

Let

$$
X=\left\{u \in W^{1,2}\left(\mathbb{R}, \mathbb{R}^{N}\right) \mid \int_{\mathbb{R}} e^{Q(t)}\left[|\dot{u}(t)|^{2}+(L(t) u(t), u(t))\right] d t<+\infty\right\},
$$

where $Q(t)$ is defined in (1.2). Then $X$ is a weighted Sobolev space with the norm given by

$$
\|u\|=\left(\int_{\mathbb{R}} e^{Q(t)}\left[|\dot{u}(t)|^{2}+(L(t) u(t), u(t))\right] d t\right)^{1 / 2}, \quad u, v \in X
$$

It is obvious that

$$
X \subset L^{2}\left(e^{Q(t)}\right)
$$


with the embedding being continuous. Here $L^{q}\left(e^{Q(t)}\right)(2 \leq q<+\infty)$ denotes the Banach spaces of functions on $\mathbb{R}$ with values in $\mathbb{R}^{N}$ under the norm

$$
\|u\|_{q}:=\left\{\int_{\mathbb{R}} e^{Q(t)}|u(t)|^{q} d t\right\}^{1 / q} .
$$

If $\sigma$ is a positive, continuous function on $\mathbb{R}$ and $1<s<+\infty$, let

$$
L_{\sigma}^{s}\left(e^{Q(t)}\right)=\left\{\left.u \in L_{\mathrm{loc}}^{1}\left(e^{Q(t)}\right)\left|\int_{\mathbb{R}} \sigma(t) e^{Q(t)}\right| u(t)\right|^{s} d t<+\infty\right\} .
$$

$L_{\sigma}^{s}$ equipped with the norm

$$
\|u\|_{s, \sigma}=\left(\int_{\mathbb{R}} \sigma(t) e^{Q(t)}|u(t)|^{s} d t\right)^{1 / s}
$$

is a reflexive Banach space.

Set $E=X \cap L_{a}^{p}\left(e^{Q(t)}\right)$, where $a$ is the function given in condition (A). Then $E$ with its standard norm $\|\cdot\|$ is a reflexive Banach space. Similar to [20-25], the definition of fast homoclinic solutions is given in the following.

Definition 1.1 If (1.2) holds, a solution of (1.1) is called a fast homoclinic solution if $u \in E$.

The main results are given in the following.

Theorem 1.1 Suppose that $q$ satisfies (1.2), $a, L$, and $W$ satisfy the following conditions:

(A) Let $p>2, a(t)$ is a continuous, positive function on $\mathbb{R}$ such that, for all $t \in \mathbb{R}$,

$$
a(t) \geq \vartheta|t|^{\beta}, \quad \vartheta>0, \beta>(p-2) / 2 .
$$

(L) $L \in C\left(\mathbb{R}, \mathbb{R}^{N} \times \mathbb{R}^{N}\right)$ is an $M^{N}(\mathbb{R})$-valued continuous function of $t \in \mathbb{R}$, and there exists a constant $\gamma>0$ such that

$$
(L(t) x, x) \geq \gamma|x|^{2}, \quad \forall(t, x) \in \mathbb{R} \times \mathbb{R}^{N}
$$

(W1) $W(t, x)=W_{1}(t, x)-W_{2}(t, x), W_{1}, W_{2} \in C^{1}\left(\mathbb{R} \times \mathbb{R}^{N}, \mathbb{R}\right)$, and there exists a constant $R>0$ such that

$$
|\nabla W(t, x)|=o\left(|x|^{p-1}\right) \quad \text { as } x \rightarrow 0
$$

uniformly in $t \in(-\infty,-R] \cup[R,+\infty)$.

(W2) There is a constant $\mu>p$ such that

$$
0<\mu W_{1}(t, x) \leq\left(\nabla W_{1}(t, x), x\right), \quad \forall(t, x) \in \mathbb{R} \times \mathbb{R}^{N} \backslash\{0\}
$$

(W3) $W_{2}(t, 0)=0$ and there exists a constant $\varrho \in(p, \mu)$ such that

$$
W_{2}(t, x) \geq 0, \quad\left(\nabla W_{2}(t, x), x\right) \leq \varrho W_{2}(t, x), \quad \forall(t, x) \in \mathbb{R} \times \mathbb{R}^{N}
$$


Then problem (1.1) has at least one nontrivial fast homoclinic solution.

Theorem 1.2 Suppose that $q, a, L$, and W satisfy (1.2), (A), (L), (W2), and the following conditions:

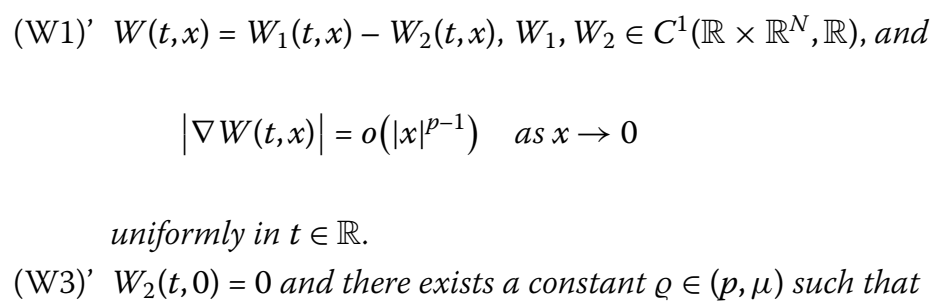

$$
\left(\nabla W_{2}(t, x), x\right) \leq \varrho W_{2}(t, x), \quad \forall(t, x) \in \mathbb{R} \times \mathbb{R}^{N}
$$

Then problem (1.1) has at least one nontrivial fast homoclinic solution.

The rest of this paper is organized as follows. In Sect. 2, some preliminaries are presented. In Sect. 3, the proofs of the main results are given. In Sect. 4, two examples are given to illustrate the main results.

\section{Preliminaries}

The functional $\varphi$ corresponding to (1.1) on $E$ is given by

$$
\begin{aligned}
\varphi(u)= & \int_{\mathbb{R}} e^{Q(t)}\left\{\frac{1}{2}\left[|\dot{u}(t)|^{2}+(L(t) u(t), u(t))\right]+\frac{a(t)}{p}|u(t)|^{p}-W(t, u(t))\right\} d t, \\
& u \in E
\end{aligned}
$$

Clearly, it follows from (A), (L), (W1), or (W1)' that $\varphi: E \rightarrow \mathbb{R}$. By Theorem 2.1 of [6], we can deduce that the map

$$
u \rightarrow a(t) e^{Q(t)}|u(t)|^{p-2} u(t)
$$

is continuous from $L_{a}^{p}\left(e^{Q(t)}\right)$ in the dual space $L_{a^{-1 /(p-1)}}^{p_{1}}\left(e^{Q(t)}\right)$, where $p_{1}=\frac{p}{p-1}$. As the embeddings $E \subset X \subset L^{\kappa}\left(e^{Q(t)}\right)$ for all $\kappa \geq 2$ are continuous, if (A), (L) and (W1) or (W1)' hold, then $\varphi \in C^{1}(E, \mathbb{R})$ and one can easily check that for $u \in E$

$$
\begin{aligned}
\left\langle\varphi^{\prime}(u), v\right\rangle= & \int_{\mathbb{R}} e^{Q(t)}\left[(\dot{u}(t), \dot{v}(t))+(L(t) u(t), v(t))+a(t)|u(t)|^{p-2}(u(t), v(t))\right. \\
& -(\nabla W(t, u(t)), v(t))] d t .
\end{aligned}
$$

Furthermore, the critical points of $\varphi$ in $E$ are classical solutions of (1.1) with $u( \pm \infty)=0$.

Let $E$ and $\|\cdot\|$ be given in Sect. 1. The following lemmas are important.

Lemma 2.1 ([1]) For any $u \in E$,

$$
\|u\|_{\infty} \leq \frac{1}{\sqrt{2 e_{0} \sqrt{\gamma}}}\|u\|=\frac{1}{\sqrt{2 e_{0} \sqrt{\gamma}}}\left\{\int_{\mathbb{R}} e^{Q(s)}\left[|\dot{u}(s)|^{2}+(L(s) u(s), u(s))\right] d s\right\}^{1 / 2},
$$




$$
|u(t)| \leq \frac{1}{\sqrt[4]{\gamma}}\left\{\int_{t}^{+\infty} e^{-Q(s)} e^{Q(s)}\left[|\dot{u}(s)|^{2}+(L(s) u(s), u(s))\right] d s\right\}^{1 / 2}
$$

and

$$
|u(t)| \leq \frac{1}{\sqrt[4]{\gamma}}\left\{\int_{-\infty}^{t} e^{-Q(s)} e^{Q(s)}\left[|\dot{u}(s)|^{2}+(L(s) u(s), u(s))\right] d s\right\}^{1 / 2},
$$

where $\|u\|_{\infty}=\operatorname{ess}_{\sup }|t \in \mathbb{R}| u(t) \mid, e_{0}=e^{\min \{Q(t): t \in \mathbb{R}\}}$.

Lemma 2.2 ([24]) If a satisfies assumption (A), then

the embedding $L_{a}^{p}\left(e^{Q(t)}\right) \subset L^{2}\left(e^{Q(t)}\right)$ is continuous.

Moreover, there exists a Hilbert space Z such that

the embeddings $L_{a}^{p}\left(e^{Q(t)}\right) \subset Z \subset L^{2}\left(e^{Q(t)}\right)$ are continuous,

the embedding $X \cap Z \subset L^{2}\left(e^{Q(t)}\right)$ is compact.

The following lemma is the mountain pass theorem which is very useful in the proofs of our theorems.

Lemma 2.3 ([29]) Let E be a real Banach space and $I \in C^{1}(E, \mathbb{R})$ satisfy the (PS)-condition. Suppose $I(0)=0$ and

(i) There exist constants $\rho, \alpha>0$ such that $I_{\partial B_{\rho}(0)} \geq \alpha$.

(ii) There exists $e \in E \backslash \bar{B}_{\rho}(0)$ such that $I(e) \leq 0$.

Then I possesses a critical value $c \geq \alpha$ which can be characterized as $c=$ $\inf _{h \in \Phi} \max _{s \in[0,1]} I(h(s))$, where $\Phi=\{h \in C([0,1], E) \mid h(0)=0, h(1)=e\}$ and $B_{\rho}(0)$ is an open ball in $E$ of radius $\rho$ centered at 0 .

Lemma 2.4 Assume that (W2) and (W3) or (W3)' hold. Then, for every $(t, x) \in \mathbb{R} \times \mathbb{R}^{N}$,

(i) $s^{-\mu} W_{1}(t, s x)$ is nondecreasing on $(0,+\infty)$;

(ii) $s^{-\varrho} W_{2}(t, s x)$ is nonincreasing on $(0,+\infty)$.

The proof of Lemma 2.4 is routine and we omit it.

\section{Proofs of theorems}

Proof of Theorem 1.1 The proof of Theorem 1.1 is divided into three steps. Step 1. We will prove that the functional $\varphi$ satisfies the (PS)-condition. Let $\left\{u_{n}\right\} \subset E$ satisfying $\varphi\left(u_{n}\right)$ be bounded and $\varphi^{\prime}\left(u_{n}\right) \rightarrow 0$ as $n \rightarrow \infty$. Then there exists a constant $C_{1}>0$ such that

$$
\left|\varphi\left(u_{n}\right)\right| \leq C_{1}, \quad\left\|\varphi^{\prime}\left(u_{n}\right)\right\|_{E^{*}} \leq \mu C_{1} .
$$

From (2.1), (2.2), (3.1), (W2), and (W3), we have

$$
2 C_{1}+2 C_{1}\left\|u_{n}\right\| \geq 2 \varphi\left(u_{n}\right)-\frac{2}{\mu}\left\langle\varphi^{\prime}\left(u_{n}\right), u_{n}\right\rangle
$$




$$
\begin{aligned}
= & \frac{\mu-2}{\mu}\left\|u_{n}\right\|^{2}+\left(\frac{2}{p}-\frac{2}{\mu}\right) \int_{\mathbb{R}} e^{Q(t)} a(t)\left|u_{n}(t)\right|^{p} d t \\
& -2 \int_{\mathbb{R}} e^{Q(t)}\left[W_{1}\left(t, u_{n}(t)\right)-\frac{1}{\mu}\left(\nabla W_{1}\left(t, u_{n}(t)\right), u_{n}(t)\right)\right] d t \\
& +2 \int_{\mathbb{R}} e^{Q(t)}\left[W_{2}\left(t, u_{n}(t)\right)-\frac{1}{\mu}\left(\nabla W_{2}\left(t, u_{n}(t)\right), u_{n}(t)\right)\right] d t \\
\geq & \frac{\mu-2}{\mu}\left\|u_{n}\right\|^{2}+\left(\frac{2}{p}-\frac{2}{\mu}\right)\left\|u_{n}\right\|_{p, a}^{p} .
\end{aligned}
$$

It follows from Lemma 2.2, $\mu>p>2$, and the above inequalities that there exists a constant $C_{2}>0$ such that

$$
\left\|u_{n}\right\| \leq C_{2}, \quad n \in \mathbb{N}
$$

Now we prove that $u_{n} \rightarrow u_{0}$ in $E$. Passing to a subsequence if necessary, we can assume that $u_{n} \rightarrow u_{0}$ in $E$. For any given number $\varepsilon>0$, from (W1), we can choose $\xi>0$ such that

$$
|\nabla W(t, x)| \leq \varepsilon \gamma|x|^{p-1} \quad \text { for }|t| \geq R \text { and }|x| \leq \xi
$$

Since $Q(t) \rightarrow \infty$ as $|t| \rightarrow \infty$, we can take $T>R$ such that

$$
Q(t) \geq \ln \left(\frac{C_{2}^{2}}{\sqrt{\gamma} \xi^{2}}\right) \text { for }|t| \geq T
$$

It follows from (2.4), (3.2), and (3.4) that

$$
\begin{aligned}
\left|u_{n}(t)\right|^{2} & \leq \frac{1}{\sqrt{\gamma}} \int_{t}^{+\infty} e^{-Q(s)} e^{Q(s)}\left[\left|\dot{u}_{n}(s)\right|^{2}+\left(L(s) u_{n}(s), u_{n}(s)\right)\right] d s \\
& \leq \frac{\xi^{2}}{C_{2}^{2}}\left\|u_{n}\right\|^{2} \leq \xi^{2} \quad \text { for } t \geq T \text { and } n \in \mathbb{N} .
\end{aligned}
$$

Similarly, from (2.5), (3.2), and (3.4), we have

$$
\left|u_{n}(t)\right|^{2} \leq \xi^{2} \quad \text { for } t \leq-T \text { and } n \in \mathbb{N} \text {. }
$$

Since $u_{n} \rightarrow u_{0}$ in $E$, it is easy to verify that $u_{n}(t)$ converges to $u_{0}(t)$ pointwise for all $t \in \mathbb{R}$. Hence, it follows from (3.5) and (3.6) that

$$
\left|u_{0}(t)\right| \leq \xi \quad \text { for } t \in(-\infty,-T] \cup[T,+\infty)
$$

Since $e^{Q(t)} \geq e_{0}>0$ on $[-T, T]=J$, the operator defined by $S: E \rightarrow H^{1}(J):\left.u \rightarrow u\right|_{J}$ is a linear continuous map. Hence $u_{n} \rightarrow u_{0}$ in $H^{1}(J)$. The Sobolev theorem implies that $u_{n} \rightarrow$ $u_{0}$ uniformly on $J$, hence there is $n_{0} \in \mathbb{N}$ such that

$$
\int_{-T}^{T} e^{Q(t)}\left|\nabla W\left(t, u_{n}(t)\right)-\nabla W\left(t, u_{0}(t)\right)\right|\left|u_{n}(t)-u_{0}(t)\right| d t<\varepsilon \quad \text { for } n \geq n_{0}
$$


It follows from (L), (3.2), (3.3), (3.5), (3.6), (3.7), and Young's inequality that

$$
\begin{aligned}
& \int_{\mathbb{R} \backslash[-T, T]} e^{Q(t)}\left|\nabla W\left(t, u_{n}(t)\right)-\nabla W\left(t, u_{0}(t)\right)\right|\left|u_{n}(t)-u_{0}(t)\right| d t \\
& \quad \leq \int_{\mathbb{R} \backslash[-T, T]} e^{Q(t)}\left(\left|\nabla W\left(t, u_{n}(t)\right)\right|+\left|\nabla W\left(t, u_{0}(t)\right)\right|\right)\left(\left|u_{n}(t)\right|+\left|u_{0}(t)\right|\right) d t \\
& \quad \leq \varepsilon \int_{\mathbb{R} \backslash[-T, T]} e^{Q(t)} \gamma\left(\left|u_{n}(t)\right|^{p-1}+\left|u_{0}(t)\right|^{p-1}\right)\left(\left|u_{n}(t)\right|+\left|u_{0}(t)\right|\right) d t \\
& \quad \leq 2 \varepsilon \int_{\mathbb{R} \backslash[-T, T]} e^{Q(t)} \gamma\left(\left|u_{n}(t)\right|^{p}+\left|u_{0}(t)\right|^{p}\right) d t \\
& \quad \leq 2 \xi^{p-2} \varepsilon \int_{\mathbb{R} \backslash[-T, T]} e^{Q(t)} \gamma\left(\left|u_{n}(t)\right|^{2}+\left|u_{0}(t)\right|^{2}\right) d t \\
& \quad \leq 2 \xi^{p-2} \varepsilon \int_{\mathbb{R} \backslash[-T, T]} e^{Q(t)}\left[\left(L(t) u_{n}(t), u_{n}(t)\right)+\left(L(t) u_{0}(t), u_{0}(t)\right)\right] d t \\
& \leq 2 \xi^{p-2} \varepsilon\left(\left\|u_{n}\right\|^{2}+\left\|u_{0}\right\|^{2}\right) \\
& \leq 2 \xi^{p-2} \varepsilon\left(C_{2}^{2}+\left\|u_{0}\right\|^{2}\right), \quad n \in \mathbb{N} .
\end{aligned}
$$

It follows from (3.8) and (3.9) that

$$
\int_{\mathbb{R}} e^{Q(t)}\left|\nabla W\left(t, u_{n}(t)\right)-\nabla W\left(t, u_{0}(t)\right)\right|\left|u_{n}(t)-u_{0}(t)\right| d t \rightarrow 0 \quad \text { as } n \rightarrow \infty .
$$

From (2.2), as $n \rightarrow \infty$, we have

$$
\begin{aligned}
0 \leftarrow & \left.\left\langle\varphi^{\prime}\left(u_{n}\right)-\varphi^{\prime}\left(u_{0}\right), u_{n}-u_{0}\right)\right\rangle \\
= & \left\|u_{n}-u_{0}\right\|^{2}+\int_{\mathbb{R}} e^{Q(t)} a(t)\left(\left|u_{n}(t)\right|^{p-2} u_{n}(t)-\left|u_{0}(t)\right|^{p-2} u_{0}(t)\right)\left(u_{n}(t)-u_{0}(t)\right) d t \\
& -\int_{\mathbb{R}} e^{Q(t)}\left(\nabla W\left(t, u_{n}(t)\right)-\nabla W\left(t, u_{0}(t)\right), u_{n}(t)-u_{0}(t)\right) d t .
\end{aligned}
$$

It is easy to see that, for any $\varsigma>1$, there exists a constant $C_{3}>0$ such that

$$
\left(|x|^{\varsigma-1} x-|y|^{\varsigma^{-1}} y\right)(x-y) \geq C_{3}|x-y|^{\varsigma+1}, \quad \forall x, y \in \mathbb{R} .
$$

Hence, there exists a constant $C_{4}>0$ such that

$$
\begin{aligned}
& \int_{\mathbb{R}} e^{Q(t)} a(t)\left(\left|u_{n}(t)\right|^{p-2} u_{n}(t)-\left|u_{0}(t)\right|^{p-2} u_{0}(t)\right)\left(u_{n}(t)-u_{0}(t)\right) d t \\
& \quad \geq C_{4} \int_{\mathbb{R}} e^{Q(t)} a(t)\left|u_{n}(t)-u_{0}(t)\right|^{p} d t .
\end{aligned}
$$

It follows from (3.10), (3.11), and (3.13) that

$$
\left\|u_{n}\right\| \rightarrow\left\|u_{0}\right\| \quad \text { as } n \rightarrow \infty
$$

and

$$
\int_{\mathbb{R}} e^{Q(t)} a(t)\left|u_{n}(t)\right|^{p} d t \rightarrow \int_{\mathbb{R}} e^{Q(t)} a(t)\left|u_{0}(t)\right|^{p} d t \quad \text { as } n \rightarrow \infty .
$$


Hence, it follows from (3.14) and (3.15) that $u_{n} \rightarrow u_{0}$ in $E$. This shows that $\varphi$ satisfies the (PS)-condition.

Step 2. From (W1), there exists $\delta \in(0,1)$ such that

$$
|\nabla W(t, x)| \leq \frac{\gamma}{2}|x|^{p-1} \quad \text { for }|t| \geq R,|x| \leq \delta .
$$

By (3.16), we have

$$
|W(t, x)| \leq \frac{\gamma}{2 p}|x|^{p} \quad \text { for }|t| \geq R,|x| \leq \delta
$$

Let

$$
C_{5}=\sup \left\{\frac{W_{1}(t, x)}{\gamma}|t \in[-R, R], x \in \mathbb{R},| x \mid=1\right\} .
$$

Set $\sigma=\min \left\{1 /\left(2 p C_{5}+1\right)^{1 /(\mu-2)}, \delta\right\}$ and $\|u\|=\sqrt{2 e_{0} \sqrt{\gamma}} \sigma:=\rho$, it follows from Lemma 2.1 that $|u(t)| \leq \sigma \leq \delta<1$ for $t \in \mathbb{R}$. From Lemma 2.4(i), (L), and (3.18), we have

$$
\begin{aligned}
\int_{-R}^{R} e^{Q(t)} W_{1}(t, u(t)) d t & \leq \int_{\{t \in[-R, R]: u(t) \neq 0\}} e^{Q(t)} W_{1}\left(t, \frac{u(t)}{|u(t)|}\right)|u(t)|^{\mu} d t \\
& \leq C_{5} \gamma \int_{-R}^{R} e^{Q(t)}|u(t)|^{\mu} d t \\
& \leq C_{5} \sigma^{\mu-2} \int_{-R}^{R} e^{Q(t)} \gamma|u(t)|^{2} d t \\
& \leq C_{5} \sigma^{\mu-2} \int_{-R}^{R} e^{Q(t)}(L(t) u(t), u(t)) d t \\
& \leq \frac{1}{2 p} \int_{-R}^{R} e^{Q(t)}(L(t) u(t), u(t)) d t .
\end{aligned}
$$

By Lemma 2.2, (L), (W3), (3.17), and (3.19), we have

$$
\begin{aligned}
\varphi(u)= & \frac{1}{2}\|u\|^{2}+\frac{1}{p} \int_{\mathbb{R}} e^{Q(t)} a(t)|u(t)|^{p} d t-\int_{\mathbb{R}} e^{Q(t)} W(t, u(t)) d t \\
\geq & \frac{1}{2}\|u\|^{2}+\frac{1}{p}\|u(t)\|_{p, a}^{p}-\int_{\mathbb{R} \backslash[-R, R]} e^{Q(t)} W(t, u(t)) d t-\int_{-R}^{R} e^{Q(t)} W_{1}(t, u(t)) d t \\
\geq & \frac{1}{2}\|u\|^{2}+\frac{C_{6}}{p}\|u\|_{2}^{p}-\frac{1}{2 p} \int_{\mathbb{R} \backslash[-R, R]} \gamma e^{Q(t)}|u(t)|^{p} d t-\frac{1}{2 p} \int_{-R}^{R} e^{Q(t)}(L(t) u(t), u(t)) d t \\
\geq & \frac{1}{2}\|u\|^{2}+\frac{C_{6}}{p}\|u\|_{2}^{p}-\frac{\delta^{p-2}}{2 p} \int_{\mathbb{R} \backslash[-R, R]} e^{Q(t)}(L(t) u(t), u(t)) d t \\
& -\frac{1}{2 p} \int_{-R}^{R} e^{Q(t)}(L(t) u(t), u(t)) d t \\
\geq & \frac{1}{2}\|u\|^{2}+\frac{C_{6}}{p}\|u\|_{2}^{p}-\frac{1}{2 p} \int_{\mathbb{R} \backslash[-R, R]} e^{Q(t)}(L(t) u(t), u(t)) d t \\
& -\frac{1}{2 p} \int_{-R}^{R} e^{Q(t)}(L(t) u(t), u(t)) d t
\end{aligned}
$$




$$
\begin{aligned}
& =\frac{1}{2}\|u\|^{2}+\frac{C_{6}}{p}\|u\|_{2}^{p}-\frac{1}{2 p} \int_{\mathbb{R}} e^{Q(t)}(L(t) u(t), u(t)) d t \\
& \geq \frac{p-1}{2 p}\|u\|^{2},
\end{aligned}
$$

where $C_{6}$ is a positive constant. Therefore, we can choose a constant $\alpha>0$ depending on $\rho$ such that $\varphi(u) \geq \alpha$ for any $u \in E$ with $\|u\|=\rho$.

Step 3. From Lemma 2.4(ii) and (2.3), we have, for any $u \in E$,

$$
\begin{aligned}
& \int_{-4}^{4} e^{Q(t)} W_{2}(t, u(t)) d t \\
& \quad=\int_{\{t \in[-4,4]:|u(t)|>1\}} e^{Q(t)} W_{2}(t, u(t)) d t+\int_{\{t \in[-4,4]:|u(t)| \leq 1\}} e^{Q(t)} W_{2}(t, u(t)) d t \\
& \quad \leq \int_{\{t \in[-4,4]:|u(t)|>1\}} e^{Q(t)} W_{2}\left(t, \frac{u(t)}{|u(t)|}\right)|u(t)|^{\varrho} d t+\int_{-4}^{4} e^{Q(t)} \max _{|x| \leq 1} W_{2}(t, x) d t \\
& \quad \leq\|u\|_{\infty}^{\varrho} \int_{-4}^{4} e^{Q(t)} \max _{|x|=1} W_{2}(t, x) d t+\int_{-4}^{4} e^{Q(t)} \max _{|x| \leq 1} W_{2}(t, x) d t \\
& \quad \leq\left(\frac{1}{\sqrt{2 e_{0} \sqrt{\gamma}}}\right)^{\varrho}\|u\|^{\varrho} \int_{-4}^{4} e^{Q(t)} \max _{|x|=1} W_{2}(t, x) d t+\int_{-4}^{4} e^{Q(t)} \max _{|x| \leq 1} W_{2}(t, x) d t \\
& =C_{7}\|u\|^{\varrho}+C_{8},
\end{aligned}
$$

where $C_{7}=\left(\frac{1}{\sqrt{2 e_{0} \sqrt{\gamma}}}\right)^{\varrho} \int_{-4}^{4} e^{Q(t)} \max _{|x|=1} W_{2}(t, x) d t, C_{8}=\int_{-4}^{4} e^{Q(t)} \max _{|x| \leq 1} W_{2}(t, x) d t$. Take $\omega \in E$ such that

$$
|\omega(t)|= \begin{cases}1 & \text { for }|t| \leq 2 \\ 0 & \text { for }|t| \geq 4\end{cases}
$$

and $|\omega(t)| \leq 1$ for $|t| \in(2,4]$. For $s>1$, from Lemma 2.4(i) and (3.21), we get

$$
\int_{-2}^{2} e^{Q(t)} W_{1}(t, s \omega(t)) d t \geq s^{\mu} \int_{-2}^{2} e^{Q(t)} W_{1}(t, \omega(t)) d t=C_{9} s^{\mu},
$$

where $C_{9}=\int_{-2}^{2} e^{Q(t)} W_{1}(t, \omega(t)) d t>0$. From (W3), (2.1), (3.20), (3.21), and (3.22), we get for $s>1$

$$
\begin{aligned}
\varphi(s \omega) & =\frac{s^{2}}{2}\|\omega\|^{2}+\frac{s^{p}}{p} \int_{\mathbb{R}} e^{Q(t)} a(t)|\omega(t)|^{p} d t+\int_{\mathbb{R}} e^{Q(t)}\left[W_{2}(t, s \omega(t))-W_{1}(t, s \omega(t))\right] d t \\
& \leq \frac{s^{2}}{2}\|\omega\|^{2}+\frac{s^{p}}{p}\|\omega\|_{p, a}^{p}+\int_{-4}^{4} e^{Q(t)} W_{2}(t, s \omega(t)) d t-\int_{-2}^{2} e^{Q(t)} W_{1}(t, s \omega(t)) d t \\
& \leq \frac{s^{2}}{2}\|\omega\|^{2}+\frac{s^{p}}{p}\|\omega\|_{p, a}^{p}+C_{7} s^{\varrho}\|\omega\|^{\varrho}+C_{8}-C_{9} s^{\mu} .
\end{aligned}
$$

Since $\mu>\varrho>p>2$ and $C_{9}>0$, it follows from (3.23) that there exists $s_{1}>1$ such that $\left\|s_{1} \omega\right\|>\rho$ and $\varphi\left(s_{1} \omega\right)<0$. Set $e=s_{1} \omega(t)$, then $e \in E,\|e\|=\left\|s_{1} \omega\right\|>\rho$ and $\varphi(e)=\varphi\left(s_{1} \omega\right)<0$. It is easy to see that $\varphi(0)=0$. From Lemma $2.3, \varphi$ has a critical value $c>\alpha$ given by

$$
c=\inf _{g \in \Phi} \max _{s \in[0,1]} \varphi(g(s))
$$


where

$$
\Phi=\{g \in C([0,1], E): g(0)=0, g(1)=e\} .
$$

Hence, there exists $u^{*} \in E$ such that

$$
\varphi\left(u^{*}\right)=c, \quad \varphi^{\prime}\left(u^{*}\right)=0 .
$$

The function $u^{*}$ is a desired solution of problem (1.1). Since $c>0, u^{*}$ is a nontrivial fast homoclinic solution. The proof is complete.

Proof of Theorem 1.2 From the proof of Theorem 1.1, we know that the condition $W_{2}(t, x) \geq 0$ in (W3) is only used in the proofs of (3.2) and Step 2. Hence, we only need to prove that (3.2) and Step 2 still hold if we use (W1)' and (W3)' instead of (W1) and (W3), respectively. We first prove that (3.2) holds. From (W2), (W3)', (2.1), (2.2), (3.1), and Lemma 2.2, we have

$$
\begin{aligned}
2 C_{1} & +\frac{2 C_{1} \mu}{\varrho}\left\|u_{n}\right\| \\
\geq & 2 \varphi\left(u_{n}\right)-\frac{2}{\varrho}\left\langle\varphi^{\prime}\left(u_{n}\right), u_{n}\right\rangle \\
= & \frac{(\varrho-2)}{\varrho}\left\|u_{n}\right\|^{2}+2 \int_{\mathbb{R}} e^{Q(t)}\left[W_{2}\left(t, u_{n}(t)\right)-\frac{1}{\varrho}\left(\nabla W_{2}\left(t, u_{n}(t)\right), u_{n}(t)\right)\right] d t \\
& -2 \int_{\mathbb{R}} e^{Q(t)}\left[W_{1}\left(t, u_{n}(t)\right)-\frac{1}{\varrho}\left(\nabla W_{1}\left(t, u_{n}(t)\right), u_{n}(t)\right)\right] d t \\
& +2\left(\frac{1}{p}-\frac{1}{\varrho}\right) \int_{\mathbb{R}} e^{Q(t)} a(t)\left|u_{n}(t)\right|^{p} d t \\
\geq & \frac{\varrho-2}{\varrho}\left\|u_{n}\right\|^{2}+2 C_{6}\left(\frac{1}{p}-\frac{1}{\varrho}\right)\left\|u_{n}\right\|_{2}^{p} .
\end{aligned}
$$

It follows from $\varrho>p>2$ and the above inequalities that there exists a constant $C_{2}>0$ such that (3.2) holds. Next, we will prove that Step 2 still holds. From (W1)', there exists $\delta \in(0,1)$ such that

$$
|\nabla W(t, x)| \leq \frac{\gamma}{2}|x|^{p-1} \quad \text { for } t \in \mathbb{R},|x| \leq \delta .
$$

By (3.25), we have

$$
|W(t, x)| \leq \frac{\gamma}{2 p}|x|^{p} \quad \text { for } t \in \mathbb{R},|x| \leq \delta .
$$

Let $\|u\|=\sqrt{2 e_{0} \sqrt{\gamma}} \delta:=\rho$, it follows from Lemma 2.1 that $|u(t)| \leq \delta$. From (L), (2.1), and (3.26) we have that

$$
\begin{aligned}
\varphi(u) & =\frac{1}{2}\|u\|+\frac{1}{p} \int_{\mathbb{R}} e^{Q(t)} a(t)|u(t)|^{p} d t-\int_{\mathbb{R}} e^{Q(t)} W(t, u(t)) d t \\
& \geq \frac{1}{2}\|u\|^{2}+\frac{1}{p}\|u\|_{p, a}^{p}-\frac{1}{2 p} \int_{\mathbb{R}} \gamma e^{Q(t)}|u(t)|^{p} d t
\end{aligned}
$$




$$
\begin{aligned}
& \geq \frac{1}{2}\|u\|^{2}+\frac{1}{p}\|u\|_{p, a}^{p}-\frac{\delta^{p-2}}{2 p} \int_{\mathbb{R}} \gamma e^{Q(t)}|u(t)|^{2} d t \\
& \geq \frac{1}{2}\|u\|^{2}+\frac{C_{6}}{p}\|u\|_{2}^{p}-\frac{\delta^{p-2}}{2 p} \int_{\mathbb{R}} e^{Q(t)}(L(t) u(t), u(t)) d t \\
& \geq \frac{1}{2}\|u\|^{2}+\frac{C_{6}}{p}\|u\|_{2}^{p}-\frac{1}{2 p} \int_{\mathbb{R}} e^{Q(t)}(L(t) u(t), u(t)) d t \\
& \geq \frac{p-1}{2 p}\|u\|^{2} .
\end{aligned}
$$

Therefore, we can choose a constant $\alpha>0$ depending on $\rho$ such that $\varphi(u) \geq \alpha$ for any $u \in E$ with $\|u\|=\rho$. The proof of Theorem 1.2 is complete.

\section{Examples}

Example 4.1 Consider the following system:

$$
\ddot{u}(t)+t \dot{u}(t)-L(t) u(t)-|t||u(t)| u(t)+\nabla W(t, u(t))=0, \quad \text { a.e. } t \in \mathbb{R},
$$

where $q(t)=t, p=3, a=|t|, t \in \mathbb{R}, u \in \mathbb{R}^{N}$. Let

$$
W(t, x)=\sum_{i=1}^{m} a_{i}|x|^{\mu_{i}}-\sum_{j=1}^{n} b_{j}|x|^{e_{j}}, \quad L(t)=\operatorname{diag}\left(1+t^{2}, \ldots, 1+t^{2}\right),
$$

where $\mu_{1}>\mu_{2}>\cdots>\mu_{m}>\varrho_{1}>\varrho_{2}>\cdots>\varrho_{n}>3, a_{i}, b_{j}>0, i=1, \ldots, m, j=1, \ldots, n$. Let

$$
W_{1}(t, x)=\sum_{i=1}^{m} a_{i}|x|^{\mu_{i}}, \quad W_{2}(t, x)=\sum_{j=1}^{n} b_{j}|x|^{\rho_{j}} .
$$

Then it is easy to check that all the conditions of Theorem 1.1 are satisfied with $\mu=\mu_{m}$ and $\varrho=\varrho_{1}$. Hence, problem (4.1) has at least one nontrivial fast homoclinic solution.

Example 4.2 Consider the following system:

$$
\ddot{u}(t)+\left(t+t^{3}\right) \dot{u}(t)-L(t) u(t)-|t|^{3}|u(t)|^{2} u(t)+\nabla W(t, u(t))=0, \quad \text { a.e. } t \in \mathbb{R},
$$

where $q(t)=t+t^{3}, p=4, a=|t|^{3}, t \in \mathbb{R}, u \in \mathbb{R}^{N}$. Let $L$ be the same in Example 4.1 and

$$
W(t, x)=a_{1}|x|^{\mu_{1}}+a_{2}|x|^{\mu_{2}}+a_{3}|x|^{\mu_{3}}-b_{1}(\sin t)|x|^{\varrho_{1}}-b_{2}(\cos t)|x|^{\varrho_{2}}-b_{3}|x|^{\varrho_{3}},
$$

where $\mu_{1}>\mu_{2}>\mu_{3}>\varrho_{1}>\varrho_{2}>\varrho_{3}>4, a_{1}, a_{2}, a_{3}>0, b_{1}, b_{2}, b_{3}>0$. Let

$$
\begin{aligned}
& W_{1}(t, x)=a_{1}|x|^{\mu_{1}}+a_{2}|x|^{\mu_{2}}+a_{3}|x|^{\mu_{3}}, \\
& W_{2}(t, x)=b_{1}(\sin t)|x|^{\varrho_{1}}+b_{2}(\cos t)|x|^{\varrho_{2}}+b_{3}|x|^{\varrho_{3}} .
\end{aligned}
$$

Then it is easy to check that all the conditions of Theorem 1.2 are satisfied with $\mu=\mu_{3}$ and $\varrho=\varrho_{1}$. Hence, problem (4.2) has at least one nontrivial fast homoclinic solution. 


\title{
5 Conclusions
}

In this paper, we study fast homoclinic solutions for a type of second-order damped vibration system. The difference from other papers is that our system has both damped vibration and $L(t) u(t)$. Besides, we consider the term $a(t)|u(t)|^{p-2} u(t)$ in the system. So the system is more general than the other papers and the results obtained are more general. From this point, our work is valued.

\author{
Acknowledgements \\ The author would like to thank the referees for their useful suggestions which have significantly improved the paper. \\ Funding \\ This work is supported by the NNSF of China (No. 11301108) and Guangxi Natural Science Foundation (No. \\ 2016GXNSFAA380082)
}

Availability of data and materials

Data sharing not applicable to this article as no datasets were generated or analyzed during the current study.

\section{Competing interests}

The author declares that she has no competing interests.

Authors' contributions

The author read and approved the final manuscript.

\section{Publisher's Note}

Springer Nature remains neutral with regard to jurisdictional claims in published maps and institutional affiliations.

Received: 28 February 2018 Accepted: 4 May 2018 Published online: 10 May 2018

\section{References}

1. Chen, P., Tang, X.H.: New existence of homoclinic orbits for a second-order Hamiltonian system. Comput. Math. Appl. 62(1), 131-141 (2011)

2. Salvatore, A.: Homoclinic orbits for a class of strictly convex Hamiltonian systems. Dyn. Syst. Appl. 6(2), 153-164 (1997)

3. Salvatore, A.: On the existence of homoclinic orbits for a second-order Hamiltonian system. Differ. Integral Equ. 10(2), 381-392 (1997)

4. Ambrosetti, A., Rabinowitz, P.: Dual variational methods in critical point theory and applications. J. Funct. Anal. 14(5), 349-381 (1973)

5. Pucci, P., Radulescu, V.: The impact of the mountain pass theory in nonlinear analysis: a mathematical survey. Boll. Unione Mat. Ital. 3(9), 543-582 (2010)

6. Benci, V., Fortunato, D.: Weighted Sobolev space and the nonlinear Dirichlet problem in unbounded domains. Ann. Mat. Pura Appl. 121, 319-336 (1979)

7. Poincaré, H.: Les Méthodes Nouvelles de la Mécanique Céleste. Gauthier-Villars, Paris (1897-1899)

8. Arias, M., Campos, J., Robles-Pres, A.M., Sanchez, L.: Fast and heteroclinic solutions for a second order ODE related to Fisher-Kolmogorov's equation. Calc. Var. Partial Differ. Equ. 21(3), 319-334 (2004)

9. Chen, C.N., Tzeng, S.Y.: Existence and multiplicity results for homoclinic orbits of Hamiltonian systems. Electron. J. Differ. Equ. 1997, 7 (1997)

10. Ding, Y.H.: Existence and multiplicity results for homoclinic solutions to a class of Hamiltonian systems. Nonlinear Anal. 25(11), 1095-1113 (1995)

11. Izydorek, M., Janczewska, J.: Homoclinic solutions for a class of the second-order Hamiltonian systems. J. Differ. Equ. 219(2), 375-389 (2005)

12. Korman, P., Lazer, A.C., Li, Y.: On homoclinic and heteroclinic orbits for Hamiltonian systems. Differ. Integral Equ. 10(2), 357-368 (1997)

13. Lu, Y.F., Li, C.Y., Zhong, S.Z., Zhang, W.J.: Homoclinic orbits for a class of Hamiltonian systems with potentials changing sign. Ann. Differ. Equ. 21(3), 370-372 (2005)

14. Li, X., Wu, X.: On a class of damped vibration problems with super-quadratic potentials. Nonlinear Anal. 72(1), $135-142(2010)$

15. Rabinowitz, P.H.: Periodic and heteroclinic orbits for a periodic Hamiltonian systems. Ann. Inst. Henri Poincaré, Anal. Non Linéaire 6(5), 331-346 (1989)

16. Tang, X.H., Lin, X.Y.: Existence of infinitely many homoclinic orbits in Hamiltonian systems. Proc. R. Soc. Edinb., Sect. A, Math. 141(5), 1103-1119 (2011)

17. Wu, D.L., Wu, X.P., Tang, C.: Homoclinic solutions for a class of nonperiodic and noneven second-order Hamiltonian systems. J. Math. Anal. Appl. 367(1), 154-166 (2010)

18. Zhang, Q.F., Tang, X.H.: Existence of homoclinic solutions for a class of asymptotically quadratic non-autonomous Hamiltonian systems. Math. Nachr. 285(5-6), 778-789 (2012)

19. Zhang, Q.F., Tang, X.H.: Existence of homoclinic solutions for a class of second-order non-autonomous Hamiltonian systems. Math. Slovaca 62(5), 1-12 (2012)

20. Chen, P., Tang, X.H., Agarwal, R.P.: Fast homoclinic solutions for a class of damped vibration problems. Appl. Math. Comput. 219(11), 6053-6065 (2013) 
21. Wu, X., Zhou, J.: On a class of forced vibration problems with obstacles. J. Math. Anal. Appl. 337(2), 1053-1063 (2008)

22. $\mathrm{Wu}, \mathrm{X}$. . Chen, S.X., Teng, K.: On variational methods for a class of damped vibration problems. Nonlinear Anal. 68(6), $1432-1441(2008)$

23. Zhang, Z.H., Yuan, R.: Fast homoclinic solutions for some second order non-autonomous systems. J. Math. Anal. Appl. 376(1), 51-63 (2011)

24. Zhang, Q.F., Zhang, Q.M., Tang, X.H.: Existence of fast homoclinic orbits for a class of second-order non-autonomous problems. Bound. Value Probl. 2014, 89 (2014)

25. Zhang, Q.F.: Existence and multiplicity of fast homoclinic solutions for a class of damped vibration problems with impulsive effects. Abstr. Appl. Anal. 2014, Article ID 960276 (2014)

26. Cavalcanti, M., Domingos Cavalcanti, V., Lasiecka, I., Webler, C.: Intrinsic decay rates for the energy of a nonlinear viscoelastic equation modeling the vibrations of thin rods with variable density. Adv. Nonlinear Anal. 6(2), 121-145 (2017)

27. Rzepnicki, L.: The basis property of eigenfunctions in the problem of a nonhomogeneous damped string. Opusc. Math. 37(1), 141-165 (2017)

28. Zhang, Q.F., Li, Y.: Existence and multiplicity of fast homoclinic solutions for a class of nonlinear second-order nonautonomous systems in a weighted Sobolev space. J. Funct. Spaces 2015, Article ID 495040 (2015)

29. Rabinowitz, P.H.: Minimax Methods in Critical Point Theory with Applications to Differential Equations. CBMS Regional Conf. Ser. in Math., vol. 65. American Mathematical Society, Providence (1986)

\section{Submit your manuscript to a SpringerOpen ${ }^{\circ}$ journal and benefit from:}

- Convenient online submission

- Rigorous peer review

- Open access: articles freely available online

- High visibility within the field

- Retaining the copyright to your article 